\title{
Detection of the New Ehrlichia Species Closely Related to Ehrlichia ewingii from Haemaphysalis longicornis in Yonaguni Island, Okinawa, Japan
}

\author{
Kotaro MATSUMOTO ${ }^{1}$, Toshihiko TAKEUCHI ${ }^{1}$, Naoaki YOKOYAMA ${ }^{2}$, Yoshito KATAGIRI $^{3)}$, Mamoru OOSHIRO ${ }^{4}$, \\ Satoshi ZAKIMI $^{4)}$, Gaowa ${ }^{5)}$, Fumihiko KAWAMORI ${ }^{()}$, Norio OHASHI ${ }^{5)}$ and Hisashi INOKUMA ${ }^{1) *}$ \\ ${ }^{1)}$ Department of Applied Clinical Medicine and ${ }^{2)}$ National Research Center for Protozoan Diseases, Obihiro University of Agriculture and \\ Veterinary Medicine, Obihiro, Hokkaido 080-8555, ${ }^{3)}$ Okinawa Yaeyama Livestock Hygiene Service Center, Ishigaki, Okinawa 907-0022, \\ 4) Okinawa Prefectural Institute of Animal Health, Naha, Okinawa 900-0024, ${ }^{5)}$ Laboratory of Microbiology, Department of Food and \\ Nutritional Sciences, Graduate School of Nutritional and Environmental Sciences, Global Center of Excellence Program, University of \\ Shizuoka, Shizuoka 422-8526 and ${ }^{6}$ Department of Microbiology, Shizuoka Institute of Environment and Hygiene, Shizuoka 420-8637, \\ Japan
}

(Received 7 January 2011/Accepted 14 June 2011/Published online in J-STAGE 28 June 2011)

ABSTRACT. We collected a total of 206 Haemaphysalis longicornis ticks by flagging in pastures in Yonaguni Island, Okinawa, Japan, in April 2008. Four of the 206 tick DNA samples tested were positive in a polymerase chain reaction (PCR) screening for the 16SrRNA gene of Anaplasmataceae. Partial sequences of 4 PCR products were identical to each other. Longer sequences of the 16SrRNA gene were successfully determined in 2 of the 4 tick samples, and the obtained 1,392 bp and 1,300 bp sequences revealed high similarity to the 16SrRNA gene sequences of the validated Ehrlichia species, including Ehrlichia ewingii, E. chaffeensis, and E. canis (98.3-98.6\%). We also sequenced 1,304 bp of the groEL gene from the 2 tick samples, and found that these had the highest similarity to sequences of E. ewingii (94.0-94.4\%) in the validated ehrlichial species. Based on the 16SrRNA and groEL gene sequences, the ehrlichial agents detected in this study were similar to the Ehrlichia species detected in Asia and may compose a new Ehrlichia species with other Ehrlichia species detected in Asia.

KEY WORDS: 16SrRNA, Ehrlichia, groEL, Haemaphysalis longicornis.

Ehrlichia and Anaplasma species are Gram-negative, obligate intracellular bacteria, and are reported as pathogens of emerging diseases [2, 4]. Bacteria in the genera Ehrlichia/Anaplasma are transmitted by blood-sucking arthropods, including ticks, fleas, and lice, the reservoirs of which are wild mammals such as rodents and deer [5, 15]. Recently, Ehrlichia/Anaplasma species have been detected in mammals and ticks in Japan [6, 11]. In Yonaguni Island, which is the westernmost island of Japan and lies between the East China Sea and the Pacific Ocean (Fig. 1), Anaplasma phagocytophilum and A. bovis were detected in peripheral blood samples of grazing cattle in 2006 [11]. However, no survey of ticks in Yonaguni Island for EhrlichialAnaplasma species has been performed, and the relationship between ticks and Ehlrichia/Anaplasma species in Yonaguni Island is unclear. The present study aimed to reveal how ticks were involved in the transmission of Ehlrichia/Anaplasma species by using DNA-based tools to test for the presence of Ehrlichia/Anaplasma species in ticks in Yonaguni Island.

We used flagging methods to collect a total of 206 ticks from 6 grazing pastures on Yonaguni Island in April 2008. Following tick identification, we extracted DNA from each tick using the QIAamp DNA Mini Kit (Qiagen, Hilden, Germany). Tick DNA samples were stored at $-30^{\circ} \mathrm{C}$ until use.

\footnotetext{
* Correspondence to: Inokuma, H., Department of Applied Clinical Medicine, Obihiro University of Agriculture and Veterinary Medicine, Inada-cho, Obihiro, Hokkaido 080-8555, Japan. e-mail: inokuma@obihiro.ac.jp
}

Tick DNA samples were screened by PCR for $345 \mathrm{bp}$ of the 16SrRNA gene of Anaplasmataceae using the primer pair EHR16SD and EHR16SR [12]. Sequences of positive PCR products were determined through direct sequencing by BigDye Terminator version 3.1 Cycle Sequencing Kit (Applied Biosystems, Carlsbad, CA, U.S.A.). Obtained sequences were compared to known sequences using the BLAST program (http://blast.ncbi.nlm.nih.gov/Blast.cgi).

Tick DNA samples positive for Ehrlichia/Anaplasma species were used in PCR and sequencing of nearly the full length of the 16SrRNA gene and part of the groEL gene. The 16rRNA gene was amplified with primer pairs fD1 and EHR16SR, as well as EHR16SD and Rp2, which amplify the 16SrRNA gene of Anaplasmataceae bacteria [7]. Nested PCR was used to amplify approximately $1,300 \mathrm{bp}$ of the groEL gene of Ehrlichia species. Primer pairs HS1a and HS6a as well as HS43 and HSVR were used in the 1st and 2nd PCRs, respectively [10]. Sequencing and BLAST search of PCR products were performed as described above. Phylogenetic trees based on 16SrRNA and groEL genes were constructed using the ClustalW software program [14] and MEGA4 software [13] by the neighbor-joining method. The 16SrRNA gene sequences of Yonaguni138 and Yonaguni206 were submitted to GenBank with accession numbers HQ697588 and HQ697589, respectively, and the groEL gene sequences of Yonaguni138 and Yonaguni206 were submitted with accession numbers HQ697590 and HQ697591, respectively.

All ticks, comprising 31 females and 175 nymphs, were 


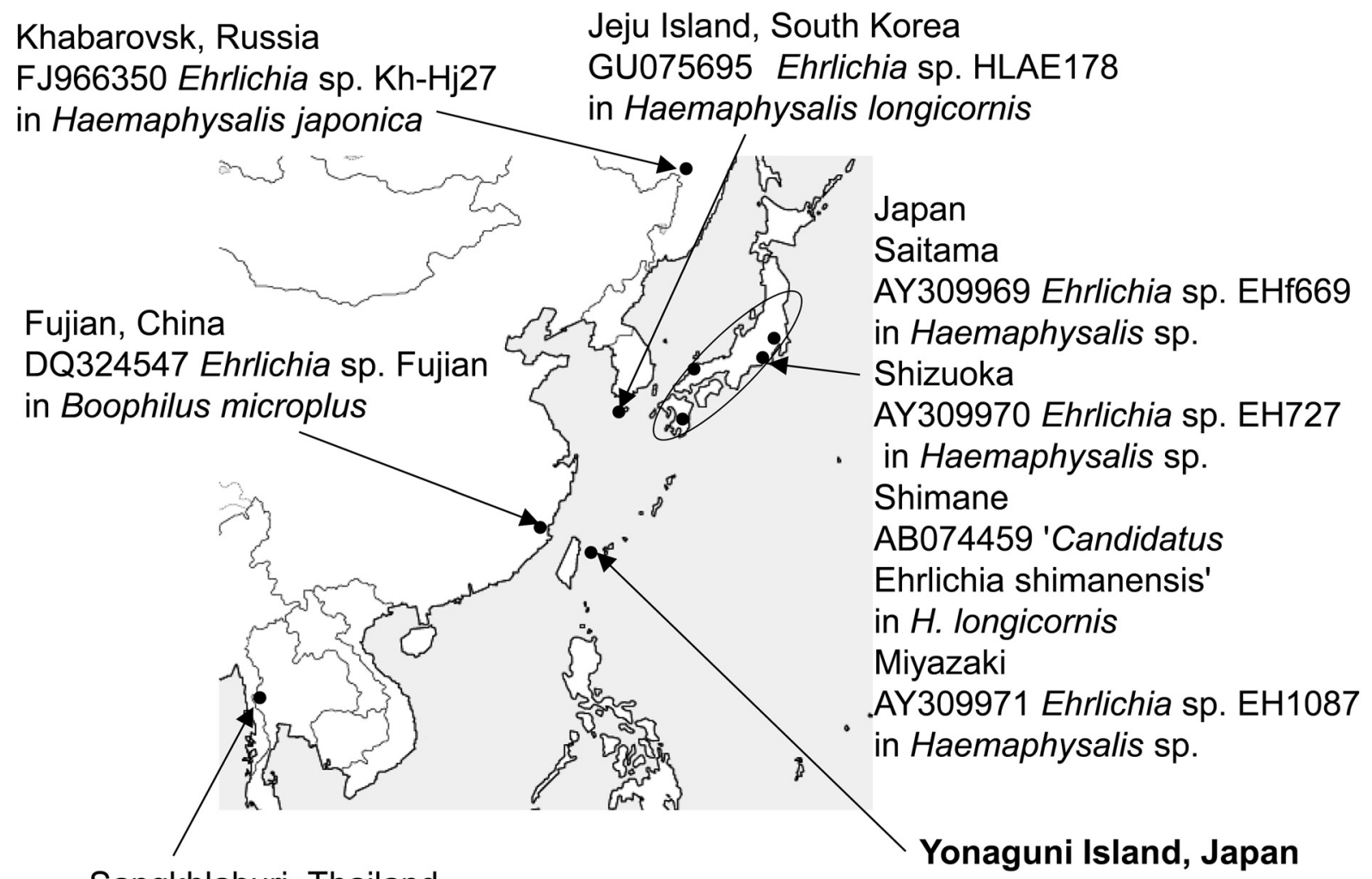

Sangkhlaburi, Thailand AF497581 Ehrlichia sp. EBm52 in B. microplus

Fig. 1. Geographical relationship of Yonaguni Island with Asian countries. The ehrlichial agents close to Ehrlichia species detected in this study, including GenBank accession numbers, tick species and country name, were shown in this figure.

identified as Haemaphysalis longicornis, which may be caused by that all ticks were collected from grazing pastures. Two females (numbers 138 and 206) and 2 nymphs of 206 tick DNA samples tested were positive in the PCR screening (1.9\%). Sequences (305 bp) of 4 positive PCR products were identical and showed high similarity to the 16SrRNA gene in Ehrlichia species. We successfully sequenced the $1,392 \mathrm{bp}$ and 1,300 bp sequences using 16SrRNA primers (numbers 138 and 206, respectively) and $1,304 \mathrm{bp}$ sequences using groEL primers in 2 of the female samples. The BLAST search for the obtained sequences using 16SrRNA primers showed the highest similarity (99.8-99.9\%) to the 16SrRNA gene of Ehrlichia sp. HLAE178 detected from H. longicornis in South Korea. The ehrlichial species with the highest similarity to sequences from Japan was Ehrlichia sp. EH727 detected in Haemaphysalis sp. in Shizuoka Prefecture (99.1\%). In the validated ehrlichial species, the 16SrRNA gene sequences of E. chaffeensis, E. canis, and E. ewingii had relatively high similarity to the obtained sequences $(98.3-98.6 \%)$. The 1,304 bp groEL sequences obtained from 2 female ticks in this study showed the highest similarity to that of Ehrlichia sp. Kh-Hj27 (94.2-94.4\%), which was detected in Haemaphysalis japonica in Khabarovsk, Russia. Our obtained sequences had the highest similarity to the groEL genes of Candidatus E. shimanensis (91.8\%) and E. ewingii (94.0$94.4 \%$ ) in the Japanese ehrlichial agents and the validated species, respectively. E. ewingii is a pathogenic agent to humans and dogs, and infection with E. ewingii causes fever, headache, thrombocytopenia, and leukopenia $[1,2]$. The ehrlichial species detected in H. longicornis from pastures on Yonaguni Island may interact with cattle. Further study of the pathogenesis and epidemiology of Ehrlichia agents is needed.

Sequences of 16SrRNA and groEL genes obtained in this study have relatively low similarity to the validated Ehrlichia species and made unique clades with other Ehrlichia species, including Ehrlichia sp. EBm52, Ehrlichia sp. HLAE178, and Ehrlichia sp. Kh-Hj27, which were detected in ticks in Thailand, South Korea, and the Russian Far East, respectively (Figs. 1, 2 and 3). These Ehrlichia species detected in Asia may represent a new Ehrlichia species. Of Ehrlichia species shown in figure 1, Ehrlichia sp. HLAE178 and 'Candidatus Ehrlichia shimanensis' were detected in $H$. longicornis, and the distribution of the ehrlchial species close to Yonaguni 138 and 206 were included in that of $H$. longicornis. Further study including isolation of the ehrlichial agent and more genetic analysis is needed. 


\section{A. phagocytophilum}

\section{E. ruminantium}
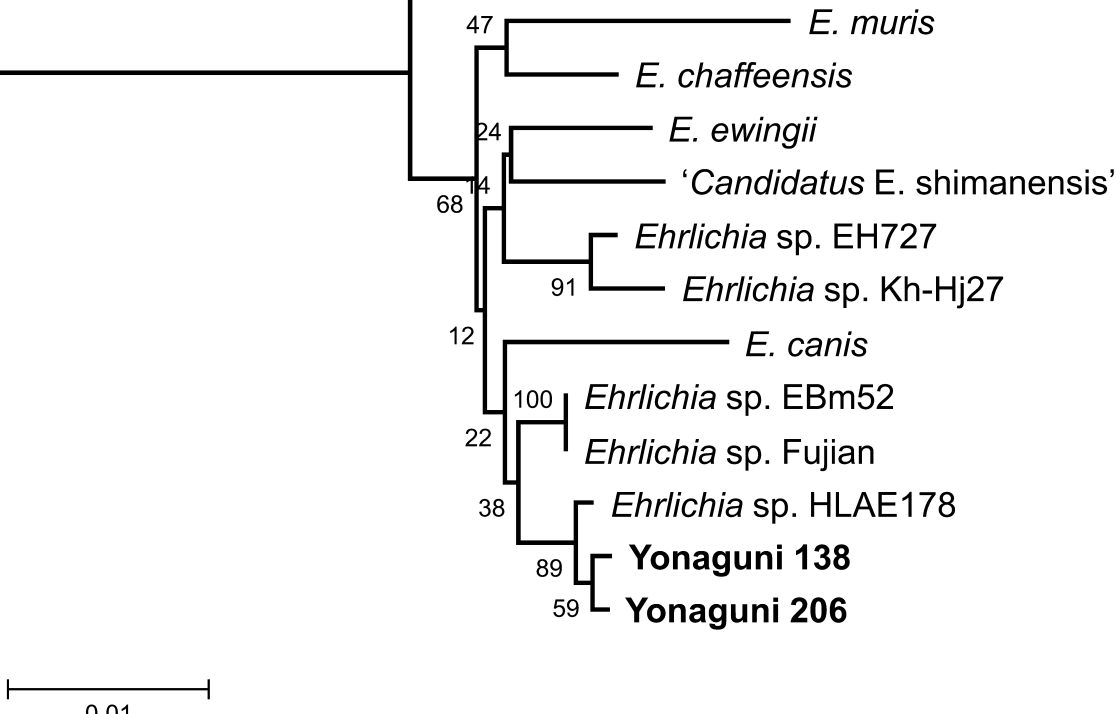

0.01

Fig. 2. Phylogenetic tree constructed by the neighbor-joining method based on sequences of the 16SrRNA gene. Yonaguni 138 and Yonaguni 206 were obtained from H. longicornis in this study. Numbers at branch nodes indicate bootstrap values. GenBank accession numbers for the sequences used to construct the trees are as follows: E. canis (M73221), E. chaffeensis (M73222), E. ewingii (U96436), E. muris (U15527), E. ruminantium (U03776), Candidatus E. shimanensis (AB074459), Ehrlichia sp. EBm52 (AF497581), Ehrlichia sp. EH727 (Y309970), Ehrlichia sp. Fujian (DQ324547), Ehrlichia sp. HLAE178 (GU075695), Ehrlichia sp. Kh-Hj27 (FJ966350), and A. phagocytophilum (CP000235).

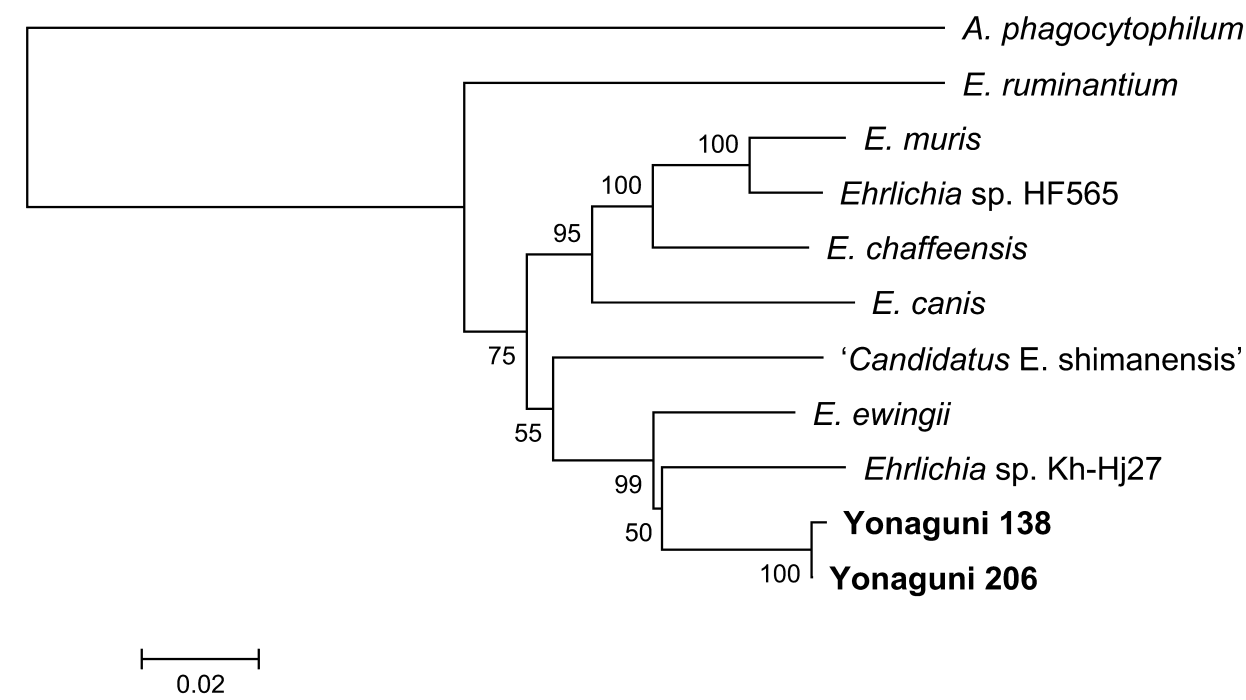

Fig. 3. Neighbor-joining tree based on the sequences of groEL gene. Yonaguni 138 and Yonaguni 206 were obtained from $H$. longicornis in the present study. Numbers at branch nodes indicate bootstrap values. GenBank accession numbers for the sequences used to construct the trees are as follows: $E$. canis (U96731), E. chaffeensis (L10917), E. ewingii (AF195273), E. muris (AF210459), E. ruminantium (DQ647014), Candidatus E. shimanensis (AB074462), Ehrlichia sp. HF565 (AB032712), Ehrlichia sp. Kh-Hj27 (FJ966349), and A. phagocytophilum (CP000235). 
An examination of blood samples from cattle on Yonaguni Island in 2006 showed a high prevalence of $A$. phagocytophilum and A. bovis [11]. However, Anaplasma species were not detected in $H$. longicornis in the present study. The grazing pastures in this study were apart from the farm surveyed previously. In South Korea, A. phagocytophilum and $A$. bovis were detected in H. longicornis [8], which suggests that $H$. longicornis can harbor these Anaplasma species. Moreover, A. phagocytophilum was detected in the rodent, Apodemus agrarius $[3,9]$. The prevalence rate of $A$. phagocytophilum in $H$. longicornis was relatively low (0$2.4 \%)[3,7,9]$, however, that in A. agrarius was high (5.6$23.6 \%$ ). The distribution of rodents in Yonaguni Island was unclear, and the rodents were not tested in this study. The absence of $A$. phagocytophilum and $A$. bovis in this study may indicate that reservoirs of $A$. phagocytophilum and $A$. bovis were not located in the studied pastures and/or that the number of tested ticks was insufficient to detect Anaplasma species.

Prevalence of Ehrlichia species of H. longicornis in this study was $1.1 \%$ (2 of 175$)$ in nymphs and $6.5 \%$ (2 of 31$)$ in adults, which may indicate that larvae and/or nymphs of $H$. longicornis become possessed of ehrlichial agents when they take blood from the hosts on the pasture on Yonaguni Island. However, the nature of the reservoir for Ehrlichia species on Yonaguni Island is not clear. Further study to reveal infection rates in mammals, including rodents, in pastures on Yonaguni Island is needed.

This study was supported in part by Grant H21-ShinkouIppan-014 for Research on Emerging and Re-Emerging Infection, a Grant-in-Aid for Scientific Research from the Japan Society for the Promotion of Science (No. 21380192).

\section{REFERENCES}

1. Anderson, B. E., Greene, C. E., Jones, D. C. and Dawson, J. E. 1992. Ehrlichia ewingii sp. nov., the etiologic agent of canine granulocytic ehrlichiosis. Int. J. Syst. Bacteriol. 42: 299-302.

2. Buller, R. S., Arens, M., Hmiel, S. P., Paddock, C. D., Sumner, J. W., Rikhisa, Y., Unver, A., Gaudreault-Keener, M., Manian, F. A., Liddell, A. M., Schmulewitz, N. and Storch, G. A. 1999. Ehrlichia ewingii, a newly recognized agent of human ehrlichiosis. New Engl. J. Med. 341: 148-155.

3. Chae, J. S., Yu do, H., Shringi, S., Klein, T. A., Kim, H. C., Chong, S. T., Lee, I. Y. and Foley, J. 2008. Microbial pathogens in ticks, rodents and a shrew in northern Gyeonggi-do near the DMZ, Korea. J. Vet. Sci. 9: 285-293.

4. Chen, S. M., Dumler, J. S., Bakken, J. S. and Walker, D. H.
1994. Identification of a granulocytotropic Ehrlichia species as the etiologic agent of human disease. J. Clin. Microbiol. 32: 589-595.

5. Des, V. F. and Fish, D. 1997. Transmission of the agent of human granulocytic ehrlichiosis by host-seeking Ixodus scapularis (Acari:Ixodidae) in southern New York state. J. Med. Entomol. 34: 379-382.

6. Inokuma, H., Beppu, T., Okuda, M., Shimada Y. and Sakata, Y. 2004. Detection of ehrlichial DNA in Haemaphysalis ticks recovered from dogs in Japan that is closely related to a novel Ehrlichia sp. found in cattle ticks from Tibet, Thailand, and Africa. J. Clin. Microbiol. 42: 1353-1355.

7. Inokuma, H., Terada, Y., Kamio, T., Raoult, D. and Brouqui, P. 2001. Analysis of the 16S rRNA gene sequence of Anaplasma centrale and its phylogenetic relatedness to other ehrlichiae. Clin. Diagn. Lab. Immunol. 8: 241-244.

8. Kim, C. M., Kim, M. S., Park, M. S., Park, J. H. and Chae, J. S. 2003. Identification of Ehrlichia chaffeensis, Anaplasma phagocytophilum, and A. bovis in Haemaphysalis longicornis and Ixodes persulcatus ticks from Korea. Vector Borne Zoonotic Dis. 3: 17-26.

9. Kim, C. M., Yi, Y. H., Yu, D. H., Lee, M. J., Cho, M. R., Desai, A. R., Shringi, S., Klein, T. A., Kim, H. C., Song, J. W., Baek, L. J., Chong, S. T., O'guinn, M .L., Lee, J. S., Lee, I. Y., Park, J. H., Foley, J. and Chae, J. S. 2006. Tick-borne rickettsial pathogens in ticks and small mammals in Korea. Appl. Environ. Microbiol. 72: 5766-5776.

10. Liz, J. S., Anderes, L., Sumner, J. W., Massung, R. F., Gern, L., Rutti, B. and Brossard, M. 2000. PCR detection of granulocytic ehrlichiae in Ixodes ricinus ticks and wild small mammals in western Switzerland. J. Clin. Microbiol. 38: 1002-1007.

11. Ooshiro, M., Zakimi, S., Matsukawa, Y., Katagiri, Y. and Inokuma, H. 2008. Detection of Anaplasma bovis and Anaplasma phagocytophilum from cattle on Yonaguni Island, Okinawa, Japan. Vet. Parasitol. 154: 360-364.

12. Parola, P., Roux, V., Camicas, J. L., Baradji, I., Brouqui, P. and Raoult, D. 2000. Detection of ehrlichiae in African ticks by polymerase chain reaction. Trans. R. Soc. Trop. Med. Hyg. 94: $707-708$.

13. Tamura, K., Dudley, J., Nei, M. and Kumar, S. 2007. MEGA4: Molecular Evolutionary Genetics Analysis (MEGA) software version 4.0. Mol. Biol. Evol. 24: 1596-1599.

14. Thompson, J. D., Higgins, D. G. and Gibson, T. J. 1994. CLUSTAL W: improving the sensitivity of progressive multiple sequence alignment through sequence weighting, positionspecific gap penalties and weight matrix choice. Nucleic Acids Res. 22: 4673-4680.

15. Yabsley, M. J., Varela, A. S., Tate, C. M., Dugan, V. G., Stallknecht, D. E., Little, S. E. and Davidson, W. R. 2002. Ehrlichia ewingii infection in white-tailed deer (Odocoileus virginianus). Emerg. Infect. Dis. 8: 668-671. 